\title{
GROUPS WHOSE PROJECTIVE CHARACTER DEGREES ARE POWERS OF A PRIME $\dagger$
}

\author{
by R. J. HIGGS
}

(Received 15 July, 1986)

1. Introduction. Let $G$ be a finite group, and $P: G \rightarrow G L(n, \mathbb{C})$ be such that for all $x, y \in G$

(i) $P(x) P(y)=\alpha(x, y) P(x y)$, and

(ii) $P(1)=I_{n}$,

where $\alpha(x, y) \in \mathbb{C}^{*}$; then $P$ is a projective representation of $G$ with cocycle $\alpha$ and degree $n$. For other basic definitions concerning projective representations see [4].

Definition 1.1. We shall say that $G$ is a $(p, \alpha)$-group if for some cocycle $\alpha$ of $G$ all the irreducible projective representations of $G$ with cocycle $\alpha$ have degree a power of the prime number $p$.

The main result of this paper is the following.

Theorem B. If $G$ is $a(p, \alpha)$-group, then $G$ is solvable.

We now review some of the known facts about $(p, 1)$-groups where 1 denotes the trivial cocycle of a group. In particular, the following theorem is well-known (see (2.3) of [5]).

Theorem A. $G$ is $a(p, 1)$-group if and only if $G$ has a normal Abelian $p$ complement.

Thus we have that Theorem B is true when $\alpha$ is the trivial cocycle of $G$. However, Theorem $\mathrm{A}$ does not hold in general when $G$ is a $(p, \alpha)$-group and $\alpha$ is not cohomologous to 1 , since $A_{4}$ provides a counter-example.

Before commencing, we remark that the degrees of projective representations are unaffected under projective equivalence, so that if $G$ is a $(p, \alpha)$-group it is also a $(p, \beta)$-group for $\beta \sim \alpha$, that is for $\beta$ cohomologous to $\alpha$. Thus in what follows it is no loss to assume that the cocycle $\alpha$ under consideration is special (see [4] for this definition). As a consequence of this we need only consider the set of irreducible projective characters of $G$ with cocycle $\alpha$, denoted by $\operatorname{Proj}(G, \alpha)$; these in particular are class functions. Also it follows from the use of a covering group and ordinary character theory that both the orthogonality relations and Frobenius' reciprocity hold for $\operatorname{Proj}(G, \alpha)$. Finally we state and use without further reference the well-known fact that $o([\alpha])$ in $H^{2}\left(G, \mathbb{C}^{*}\right)$ divides $\xi(1)$ for all $\xi \in \operatorname{Proj}(G, \alpha)$.

† This paper forms part of the author's Ph.D. thesis, Liverpool University 1985, for which support came from a SERC award.

Glasgow Math. J. 30 (1988) 177-180. 


\section{Preliminary Results.}

Defintion 2.1. Let $G$ be a group with cocycle $\alpha, N \unlhd G$, and $\zeta \in \operatorname{Proj}\left(N, \alpha_{N}\right)$. Then the $g$-conjugate $\xi^{g}$ of $\zeta$, for $g \in G$, is defined by

$$
\zeta^{g}(x)=\alpha\left(g, x g^{-1}\right) \alpha\left(x, g^{-1}\right)\left(\alpha\left(g, g^{-1}\right)\right)^{-1} \zeta\left(g x g^{-1}\right)
$$

for all $x \in N$.

It is routine to check that the above definition defines an action of $G$ on $\operatorname{Proj}\left(N, \alpha_{N}\right)$ for which Clifford's theorem as stated for ordinary characters holds. We denote the stabilizer of $\zeta$ in $G$ by $I_{G}(\zeta)$.

We now investigate the way that normal subgroups and certain quotient groups of $G$ effectively inherit the property of being $(p, \alpha)$-groups from $G$.

Lemma 2.2. Let $G$ be $a(p, \alpha)$-group, $N \unlhd G$, and $\zeta \in \operatorname{Proj}\left(N, \alpha_{N}\right)$. Then

(i) $N$ is a $\left(p, \alpha_{N}\right)$-group;

(ii) $I_{G}(\zeta) / N$ is a $(p, \beta)$-group for some cocycle $\beta$ of $I_{G}(\zeta) / N$, and $\left[G: I_{G}(\zeta)\right]$ is a power of $p$.

Proof. (i) Let $\zeta \in \operatorname{Proj}\left(N, \alpha_{N}\right)$ and $\xi \in \operatorname{Proj}(G, \alpha)$ such that $\left[\xi_{N}, \zeta\right] \neq 0$. By Clifford's theorem we have

$$
\xi_{N}=e\left(\xi_{1}+\ldots+\zeta_{t}\right)
$$

where $\zeta=\zeta_{1}, \ldots, \zeta_{t}$ are the distinct $G$-conjugates of $\zeta$, and so $\xi(1)=e t \zeta(1)$. However, since $\xi(1)$ is a power of $p$ we have that $\zeta(1)$ is also a power of $p$.

(ii) Let $T=I_{G}(\xi)$; then from Clifford's theorem there exists a cocycle $\beta$ of $T / N$ and a bijection from $\operatorname{Proj}(T / N, \beta)$ onto $\left\{\eta \in \operatorname{Proj}\left(T, \alpha_{T}\right):\left[\eta_{N}, \zeta\right] \neq 0\right\}$ defined by $\tau \mapsto \tau K$ where $\kappa \in \operatorname{Proj}\left(T, \alpha_{T} \beta^{-1}\right)$. Let $\tau \in \operatorname{Proj}(T / N, \beta)$ and $\tau \kappa=\eta$ then again by Clifford's theorem $\eta^{G}=\xi$ for $\xi \in \operatorname{Proj}(G, \alpha)$. Thus

$$
\eta^{G}(1)=[G: T] \eta(1)=[G: T] \tau(1) \kappa(1)=\xi(1)
$$

and so both $[G: T]$ and $\tau(1)$ are powers of $p$.

We have shown that, if $G$ is a $(p, \alpha)$-group, then we have some restraints on $\operatorname{Proj}\left(N, \alpha_{N}\right)$ where $N \unlhd G$. However, we can achieve very strong control over the projective characters of $G$ between $G$ and $S \in \operatorname{Syl}_{p}(G)$.

LemMa 2.3. Let $G$ be a $(p, \alpha)$-group, let $L \leq G$ be such that $p+[G: L]$ and let $\zeta \in \operatorname{Proj}\left(L, \alpha_{L}\right)$. Then $\zeta$ extends to $G$.

Proof. Let $A=\left\{\xi \in \operatorname{Proj}(G, \alpha):\left[\xi_{L}, \xi\right] \neq 0\right\}$, and $\xi_{s}$ denote an element of $A$ of smallest degree; then $\zeta(1) \leq \xi_{s}(1)$. By Frobenius' reciprocity let $\zeta^{G}=\sum_{\xi_{i} \in A} b_{i} \xi_{i}$ where $b_{i}$ is a positive integer for all $i$. Then

$$
\zeta^{G}(1)=[G: L] \zeta(1)=\sum_{\xi_{i} \in A} b_{i} \xi_{i}(1)
$$


so since $p+[G: L]$ we have

$$
(\zeta(1))_{p}=\xi_{s}(1)\left(\sum_{\xi_{i} \in A} b_{i} \xi_{i}(1)\left(\xi_{s}(1)\right)^{-1}\right)_{p}
$$

Thus $\xi_{s}(1) /(\zeta(1))_{p}$. We have shown that $\zeta(1)=\xi_{s}(1)$, and so $\zeta=\left(\xi_{s}\right)_{L}$.

We remark that 2.3 shows that $L$ is a $\left(p, \alpha_{L}\right)$-group. Finally in this section we note the following important result. Abelian.

Lemma 2.4. If $G$ is a solvable $(p, \alpha)$-group, then the Hall $p^{\prime}$-subgroups of $G$ are

Proof. This is immediate from [2, p. 245].

3. The solvability of $(p, \alpha)$-groups. In order to minimize repetition we fix the following notation for the rest of this section. Let $G$ be a non-solvable group of minimal order such that $G$ is a $(p, \alpha)$-group for some cocycle $\alpha$ of $G$, and let $S \in \operatorname{Syl}_{p}(G)$. Also if $N \leq G$ and $N \leq L \leq G$ we shall denote the group $L / N$ by $\bar{L}$. We begin by applying the results of $\$ 2$ to $G$.

Lemma 3.1. (i) $G^{\prime}=G$. (ii) If $S \leq L<G$, then $L$ is solvable and $L / L^{\prime}$ is a p-group.

Proof. (i) Suppose $G^{\prime}<G$. Then, by 2.2 (i) and the minimality of $G$, we have that $G^{\prime}$ is solvable. However it then follows that $G$ is solvable, a contradiction.

(ii) $L$ is solvable by the minimality of $G$ and 2.3. Suppose $L / L^{\prime}$ is not a $p$-group. Then $L$ has a non-trivial linear character, $\mu$, such that $L^{\prime} S \leq \operatorname{ker} \mu$, and we let $x \in L-\operatorname{ker} \mu$. Now let $\zeta \in \operatorname{Proj}\left(L, \alpha_{L}\right)$. Then by 2.3 we have that $\zeta$ and $(\mu \zeta)$ extend respectively to $\xi$ and $\xi^{\prime} \in \operatorname{Proj}(G, \alpha)$. However, since $\alpha$ is special, $\operatorname{det}(\xi)$ is a linear character of $G$ and so by (i) we must have

$$
(\operatorname{det}(\zeta))(x)=(\operatorname{det}(\xi))(x)=1
$$

but then similarly

$$
\left(\operatorname{det}\left(\xi^{\prime}\right)\right)(x)=(\operatorname{det}(\mu \zeta))(x)=(\mu(x))^{\xi(1)} \neq 1,
$$

contrary to (i).

We now show that $G$ must in fact be simple.

LemMa 3.2. $G$ is simple.

Proof. Suppose $M$ is a non-trivial maximal normal subgroup of $G$, so that $\bar{G}=G / M$ is simple by 3.1(i). Let $\zeta \in \operatorname{Proj}\left(M, \alpha_{M}\right)$ and $T=I_{G}(\zeta)$. Then by $2.2 \bar{T}$ is a solvable subgroup of $\bar{G}$ of $p$-power index, and it follows from 2.4 that $\bar{G}$ has an Abelian Hall $p^{\prime}$-subgroup $\bar{H}$. Now if $\bar{x} \in \bar{H}$ with $\bar{x} \neq \overline{1}$ we have that $\left[\bar{G}: C_{\bar{G}}(\bar{x})\right]$ is a power of $p$, which is impossible in a non-Abelian simple group. Thus no such $M$ exists.

Now in the case $p=2$, we remark without proof (for the sake of brevity) that we 
have derived enough information to show that $G$ is not a simple group via the Classification theorem, the main reduction being the facts that $2+\left|H^{2}\left(G, \mathbb{C}^{*}\right)\right|$ and that the centralizer in $G$ of a 2 -central involution is solvable. Thus a contradiction to 3.2 can be obtained.

However, for $p$ odd we can avoid the use of the Classification theorem as follows.

THEOREM 3.3. $p$ is not odd.

Proof. Suppose $p$ is odd; then by $[1$, p. 221] we have

$$
S \cap G^{\prime}=\left\langle S \cap\left(N_{G}(K)\right)^{\prime}: 1<K \text { char } S\right\rangle .
$$

Let $Z=Z(J(S))$ and $T=N_{G}(Z)$, where $J(S)$ is the Thompson subgroup of $S$. Now let $1<K$ char $S$ and $L=N_{G}(K)$, so that $S \leq L<G$. Then $L$ is solvable by 3.1(ii) and hence is $p$-constrained by [3, (6.3.3)]. Also $L$ is $p$-stable by 2.4 and $[3,(8.1 .2)]$. Thus we obtain from [3, (8.2.11)] that $L=0_{p^{\prime}}(L) N_{L}(Z)$, and so $S \cap L^{\prime}=S \cap\left(N_{L}(Z)\right)^{\prime} \leq S \cap T^{\prime}$; consequently $S \cap G^{\prime}=S \cap T^{\prime}$ since $Z$ char $S$. Finally from 3.1(ii) we have that $T / T^{\prime}$ is a non-trivial $p$-group so that $S \not T^{\prime}$, and we conclude that $S \not G^{\prime}$ contrary to 3.1(i).

We have thus established Theorem B.

\section{REFERENCES}

1. G. Glauberman, The revision project and pushing-up, pp. 207-223 in: Finite simple groups II (Academic Press, 1980).

2. D. Gluck and T. R. Wolf, Defect groups and character heights in blocks of solvable groups II, J. Algebra 87, (1984), 222-246.

3. D. Gorenstein, Finite groups, (Harper and Row, 1968).

4. R. J. Haggarty and J. F. Humphreys, Projective characters of finite groups, Proc. London Math. Soc. (3) 36 (1978), 176-192.

5. I. M. Isaacs and D. S. Passman, Groups whose irreducible representations have degrees dividing $p^{e}$, Illinois J. Math. 8 (1964), 446-457.

Department of Mathematics

UNIVERSITY COLLEGE, BelField

Dublin 4, IRELAND 